\title{
Myocardial Protection Via the Coronary Sinus Long-Term Effects of Intermittent Coronary Sinus Occlusion as an Adjunct to Reperfusion in Acute Myocardial Infarction
}

\author{
Werner Mohl, MD, PhD; Kazuo Komamura, MD ${ }^{\dagger}$; Hirofumi Kasahara, MD; \\ Georg Heinze, PhD*; Dietmar Glogar, MD, FESC**; \\ Atsushi Hirayama, MD ${ }^{\dagger+}$; Kazuhisa Kodama, MD ${ }^{\dagger \dagger}$
}

\begin{abstract}
Background Recent reports on facilitated reperfusion therapy re-address interests in coronary sinus interventions (CSI). Patients in whom short time results have been reported earlier were re-evaluated, with the aim of gathering the long-term results of pressure-controlled intermittent coronary sinus occlusion (PICSO) generated in patients with acute myocardial infarction (MI) and revascularization.

Methods and Results Thirty-four patients with ST elevated MI, in whom complete revascularization was achieved, underwent primary thrombolysis with or without PICSO. Follow-up data from these patients were collected for at least 48 months. Immediate perioperative differences were observed for time to peak creatine kinase (CK), as well as cumulative CK. In addition, the time until reperfusion was considerably less than for the control group ( $\mathrm{p}=0.014)$. Long-term data showed significant differences in reinfarction $(\mathrm{p}=0.015)$, as well as in major adverse cardiovascular events, between the 2 groups $(\mathrm{p}<0.0001)$.

Conclusion These data, because of the wide interval between collection and current analysis, could have inherited historical bias. Nonetheless, they are also uniquely indicating the potential of CSI to induce not only immediate, but also clinically significant long-term, effects as an adjunct to reperfusion therapy. Therefore, CSI should be, once again, on the study agenda and be placed under contemporary and best-available scientific scrutiny. (Circ J 2008; 72: 526-533)
\end{abstract}

Key Words: Coronary sinus interventions; Myocardial infarction; Pressure-controlled intermittent coronary sinus occlusion (PICSO)

D espite all endeavours to achieve timely reperfusion in acute coronary syndromes (ACS) many efforts fall short to limit myocardial jeopardy in the ischemia-reperfusion complex in a large cohort of patients. As seen in the current listing of the Web site clinicaltrials. gov, several interesting concepts are currently investigated to overcome this problem. Coronary sinus interventions (CSI) interfere in the coronary microcirculation using the coronary venous access. Some decades ago, during the preprimary percutaneous coronary intervention (PCI) era, CSI were a popular concept for reducing myocardial ischemia. There are 2 distinct CSI, each representing a different school of thought? There are proponents of retroperfusion of arterial blood and others proposing the concept of pres-

(Received September 25, 2007; revised manuscript received November 16, 2007; accepted November 27, 2007)

Department of Cardiothoracic Surgery, *Core Unit for Medical Statistics and Informatics Section of Clinical Biometrics, **Department of Cardiology, Medical University of Vienna, Vienna, Austria, ${ }^{\dagger} D e-$ partment of Cardiovascular Dynamics, Research Institute National Cardiovascular Center, Suita and Cardiovascular Division, Osaka Police Hospital, Osaka, Japan

The first three authors contributed equally to this work.

Mailing address: Werner Mohl, MD, PhD, Department of Cardiothoracic Surgery, Medical University of Vienna, 1090 Vienna, Waehringerguertel 18-20, Austria. E-mail: werner.mohl@meduniwien. ac.at

All rights are reserved to the Japanese Circulation Society. For permissions, please e-mail: cj@j-circ.or.jp sure-controlled intermittent coronary sinus occlusion (PICSO) methods. Syeda and colleagues reported no statistical difference in the salvage potential between these 2 methods in a meta-analysis of both methods, and showed that salvage was related to the developed pressure in the coronary sinus (CS)? Although both methods never reached widespread scientific acceptance, clinical data from a few human trials are available for both methods.,5 Retroperfusion was mainly studied in supporting coronary interventions and in cardiac surgery6,7

Today, in advancing the field of broadening the treatment options for ACS, logistic burden again makes it necessary to undo the rigid relationship between the elapsed time and potential salvage, prevent reperfusion injury, to further reduce ischemic sequelae. In addition, new pathophysiologic insight broadens our knowledge on avoidable injury and pathways on the induction of regeneration. Therefore, it seems logical to recall methods that are easily available and effective, in addition to modern revascularization strategies. Recent encouraging reports on the application of adenosine or nicorandil in addition to revascularization, ${ }^{8,9}$ or reports on stem cell-based approaches show the potential of adjunct therapies 10

It has been ignored that long-term data exist on PICSO techniques, of which short-term results have been reported earlier in this Journal. The aim of this report is to re-evaluate this concept and to analyze data on long-term results of patients with a clinically feasible 'adjunct' therapy of PICSO. 


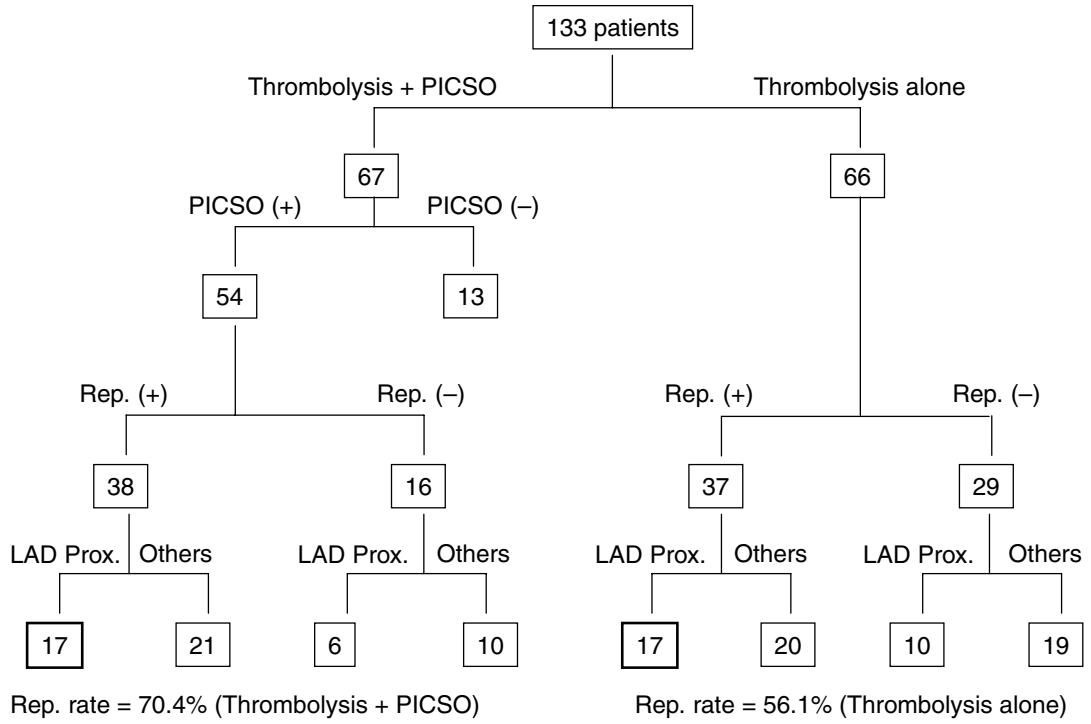

Fig 1. Flowchart of 133 patients' assignment to thrombolysis with/without pressure-controlled intermittent coronary sinus occlusion (PICSO). Two groups of patients, who obtained successful thrombolysis with a culprit lesion in the proximal left anterior descending artery (LAD), were enrolled in the study. PICSO(-), unsuccessful insertion of catheter into the coronary sinus; Rep., reperfusion; Prox., proximal.
Needless to say, this kind of study would never be collected today owing to other therapies, concepts and regimen being en vogue and, therefore, it represents a unique opportunity to evaluate pathophysiologic insight into myocardial ischemia and reperfusion, as well as pathways influencing clinical outcome.

\section{Methods}

The present study is a long-term follow up of a randomized study of patients, who underwent thrombolysis with PICSO as an adjunct therapy. Some patients in the acute phase of their disease have been reported previously, and are included in this evaluation using data collected in the late 1980s. Evaluation of data was performed in 2006.

\section{Trial Design and Patient Population}

Enrolment began in November 1985 and ended in July 1989, in Osaka Police Hospital, Osaka, Japan. During this period, 133 patients with acute myocardial infarction (AMI) underwent thrombolysis therapy, as described elsewhere? These consecutive patients were allocated randomly to 2 groups. Of 67 patients who were assigned to thrombolysis and PICSO therapy, successful catheter insertion into the CS was done in 54 patients; and 79 patients underwent thrombolysis therapy alone, as shown in Fig 1. Of these, a group of 34 patients who underwent successful thrombolysis with a culprit lesion in the proximal left anterior descending artery (LAD) was enrolled in the study. The longterm outcome of 17 patients who underwent thrombolysis plus PICSO (PICSO group) was compared with that of 17 patients who underwent thrombolysis only (control group). All patients received aspirin orally $(660 \mathrm{mg} /$ day $)$, isosorbide dinitrate ( $10 \mathrm{mg} /$ day) and diltiazem $(90 \mathrm{mg} /$ day $)$ after thrombolysis therapy. In addition, clinofibrate $(600 \mathrm{mg} /$ day $)$ was given to patients with hyperlipidemia. All patients were seen every month at the hospital's outpatient clinic.

The primary endpoint was time from discharge to first occurrence of major adverse cardiovascular events (MACE), which was defined as one of the following: reinfarction, unstable angina, PCI, coronary artery bypass grafting (CABG) or cardiac death. Secondary analyses were myocardial salvage and myocardial lactate metabolism after thrombol- ysis in the acute phase.

The study was approved by the hospital's ethics committee, and informed consent was obtained by all patients to include their data in the database. Demographic variables and clinical characteristics were recorded continuously in the department database and were extracted by retrospective review of all medical records.

\section{PICSO Instrument and Procedure}

The PICSO system consisted of an electrocardiogram and pressure monitor; pump controller; position-driven pump and a CS balloon catheter. PICSO was initiated immediately after patients were carried to the catheter laboratory, and ended after successful reperfusion was documented by angiography. Balloon inflation for $10 \mathrm{~s}$ and deflation for $5 \mathrm{~s}$ were repeated, as described elsewhere5 CS pressure was monitored continuously and gas volume in the CS balloon was optimized not to exceed $50 \mathrm{mmHg}$, according to then valid predictions about avoiding damage of coronary venous system.

\section{Evaluation for Myocardial Salvage and Myocardial Lactate Metabolism}

To detect reperfusion time electrocardiogram analysis and scoring of chest symptoms were performed every $1 \mathrm{~min}$ during thrombolysis therapy. Angiograms of the infarctrelated artery were obtained to confirm reperfusion immediately. The perfusion grade of the infarct-related artery at pre-treatment and 30 days after treatment were assessed according to the Thrombolysis In Myocardial Infarction (TIMI) study classification.1

Blood samples were collected before and just after thrombolysis. Enzymatic activity of creatine kinase (CK) was measured, as described elsewhere, and total CK release was determined using the integrated appearance function curve with the individual disappearance constant, as described elsewhere, $5,12,13$

Abnormality in wall motion was calculated by abnormally contracting segments,${ }^{14}$ global ejection fraction and regional ejection fraction, using the area method 15 from the left ventriculogram at 30 days after onset of symptoms (ie, before hospital discharge) 5 . To assess myocardial infarct size, 32 projections of single photoemission computerized 
Table 1 Baseline Patient Characteristics

\begin{tabular}{lccc}
\hline \hline & PICSO & Control & $p$ value \\
\hline Age $($ years $)$ & $60.1 \pm 13.9$ & $59.8 \pm 12.2$ & 0.94 \\
Gender $(M / F)$ & $14 / 3$ & $12 / 5$ & 0.42 \\
Hypertension & 13 & 12 & 0.7 \\
Hyperlipidemia & 7 & 4 & 0.27 \\
Diabetes mellitus & 8 & 6 & 0.49 \\
Smoking & 12 & 9 & 0.29 \\
Family history of CAD & 5 & 5 & 1 \\
Preinfarction angina & 6 & 7 & 0.73 \\
\hline
\end{tabular}

The $p$ values for all variables were not significant.

PICSO, pressure-controlled intermittent coronary sinus occlusion; $C A D$, coronary artery disease.

Table 2 Angiographical Findings at Reperfusion and 30 Days Follow up

\begin{tabular}{lccc}
\hline \hline & PICSO & Control & p value \\
\hline Reperfusion & & & \\
LAD lesion, \#6/\#7 & $9 / 8$ & $10 / 7$ & $N S$ \\
TIMI flow, grade 2/grade & $2 / 15$ & $6 / 11$ & $N S$ \\
Collaterals (+)/(-) & $2 / 15$ & $3 / 14$ & $N S$ \\
Residual stenosis & $63.8 \pm 36.7$ & $66.3 \pm 41.4$ & $N S$ \\
30 Days after reperfusion & & & $N S$ \\
TIMI flow, grade 2/grade & $2 / 15$ & $4 / 14$ & $N S$ \\
Collaterals (+)/(-) & $2 / 15$ & $4 / 14$ & $0.0126^{*}$ \\
Residual stenosis & $62.1 \pm 30$ & $77 \pm 22.6$ & 73.5 \\
Residual stenosis (BL-adjusted) & 65.5 & & \\
\hline
\end{tabular}

*Statistically significant.

LAD, left anterior descending artery; TIMI, Thrombolysis In Myocardial Infarction; BL, baseline. Other abbreviation see in Table 1.

tomography (SPECT), $3 \mathrm{~h}$ after intravenous administration of $3 \mathrm{mCi}$ of thallium-201 $(201 \mathrm{Tl})$, were obtained over $180^{\circ}$. The volume of defects was weighted for the average value of counts in the defect area, and this was defined as the extent of damaged myocardium in the total left ventricle or 'defect severity', as described elsewhere?,16,17 The QRS score was also calculated as a parameter to estimate residual infarct size during the follow-up period ${ }^{18}$

To assess lactate extraction, blood sampling was taken from the aorta and CS before and after thrombolysis was performed; and every $6 \mathrm{~h}$ for up to $24 \mathrm{~h}$ from the onset of symptoms, as described elsewhere?

\section{Statistical Analysis}

Continuous data are presented as the mean value \pm standard deviation. Baseline data were compared by means of the chi-squared test for categorical variables and t-test for continuous variables. Cardiac event-free survival curves were constructed by the Kaplan-Meier method. Statistical differences between curves were assessed using an exact log-rank test, adjusting for potentially unequal follow-up periods in the groups 19 Changes in lactate extraction ratio (LER) after reperfusion were compared between the PICSO and control groups by means of repeated measures analysis of covariance (ANCOVA), including the baseline LER as a covariate. The effect of PICSO on cardiac event-free survival was assessed by means of a Cox regression model, stating relative risk estimates and $95 \%$ confidence intervals (CI) $)^{20}$

Despite randomization, the crude effect of PICSO on event-free survival could be the result of different exposure to risk factors in the 2 groups. Hence, ideally, one would use a multivariable model that included all potential risk factors at the same time, which would adjust for such different exposure; however, because of the low total number of events in our data, such a model could not be used. Therefore, separate bivariable Cox regression models, each including group (ie, PICSO or control) and one of the risk factors (ie, age ( $\geq 65$ years or $<65$ years), gender, hypertension, hyperlipidemia, diabetes mellitus, smoking, family history, pain-to-admission time and TIMI flow), were estimated. We also estimated bivariable Cox models, including group (ie, PICSO or control) and stenosis value at Day 30 to obtain an estimate of the effect of PICSO on long-term outcome that is independent of PICSO's effect on restenosis. A p-value of $<0.05$ was considered statistically significant. Statistical analysis was performed using the SAS System, version 9.1 (SAS Institute Inc, Cary, NC, USA).

Stenosis values at Day 30 were compared between the groups, using ANCOVA, ${ }^{21}$ adjusting for differences in the baseline stenosis value at Day 0 by including the individual baseline values as a covariate. ANCOVA was also used to adjust for differences in the LER between groups for baseline differences. Average baseline-adjusted stenosis values were computed using the LSMEANS statement of SAS's PROC GLM (The SAS System, version 9.1, SAS Institute Inc). This analysis was repeated, replacing stenosis values by their ranks to obtain a confirmative non-parametric $p$-value.

\section{Results}

Baseline Patient Characteristics

Table 1 lists baseline clinical characteristics of the 2 groups. No significant difference was observed in the baseline patient characteristics and in angiographical findings just after reperfusion (Table 2). The PICSO and control group were comparable in age, gender, risk factors, site of thrombus, residual stenosis just after thrombolysis, and extent of coronary artery disease. 
Table 3 Hemodynamic Parameters in the PICSO and Control Group During Thrombolysis

\begin{tabular}{|c|c|c|c|c|}
\hline & Pre-PICSO & Post-PICSO & Control & $p$ value \\
\hline$H R$ (beats/min) & $82 \pm 17$ & $79 \pm 11$ & $85 \pm 19$ & $N S$ \\
\hline $\mathrm{AoP}(\mathrm{mmHg})$ & $130 \pm 20 / 80 \pm 13$ & $127 \pm 12 / 79 \pm 11$ & $129 \pm 20 / 75 \pm 19$ & $N S$ \\
\hline AoP (mean) & $(98 \pm 13)$ & $(95 \pm 11)$ & $(93 \pm 18)$ & $N S$ \\
\hline$P A P(m m H g)$ & $22 \pm 5 / 10 \pm 5$ & $21 \pm 5$ & $22 \pm 5 / 10 \pm 4$ & NS \\
\hline$P A P($ mean $)$ & $(14 \pm 5)$ & $(14 \pm 4)$ & $(14 \pm 5)$ & NS \\
\hline$C I\left(L \cdot \mathrm{min}^{-1} \cdot \mathrm{m}^{-2}\right)$ & $3.3 \pm 0.7$ & $3.2 \pm 0.7$ & $3.2 \pm 0.7$ & $N S$ \\
\hline
\end{tabular}

$H R$, heart rate; AoP, arterial pressure; PAP, pulmonary artery pressure; $C I$, cardiac index. Other abbreviation see in Table 1.
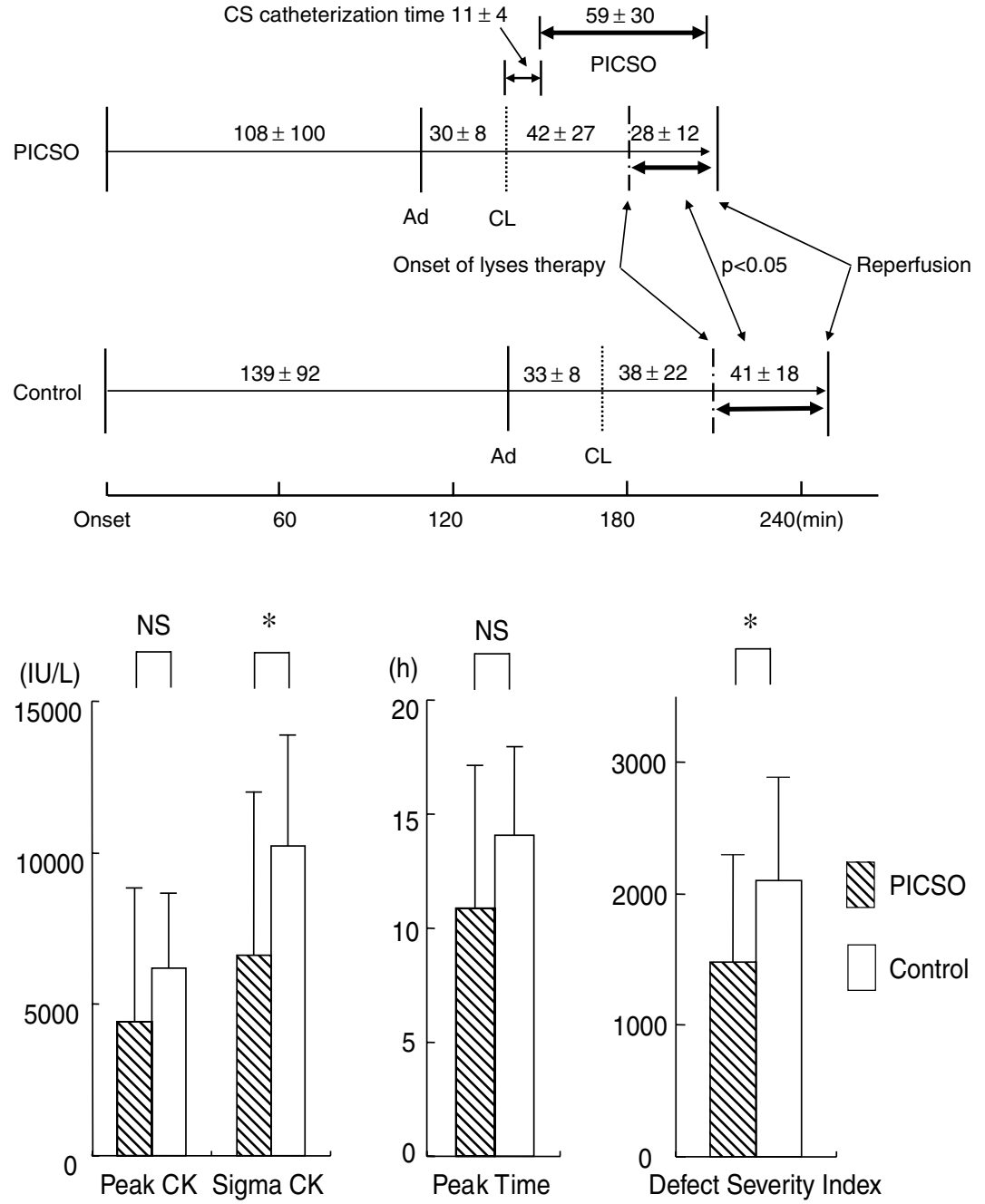
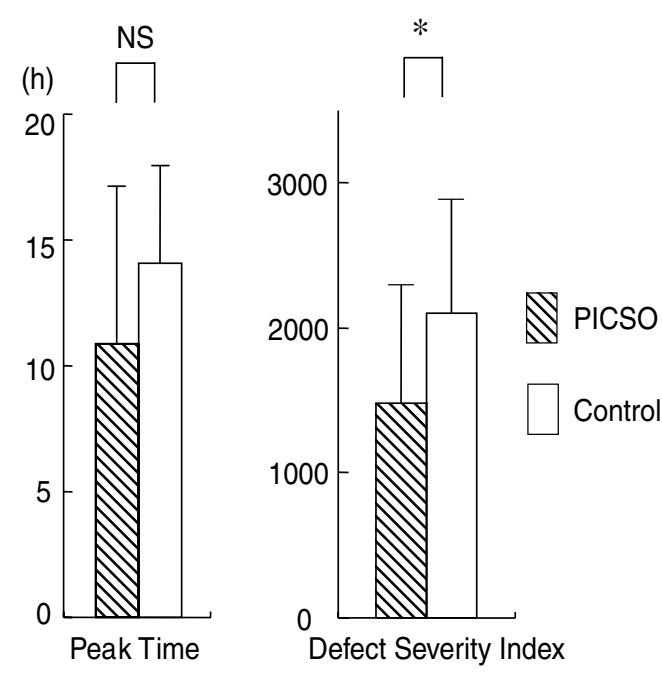

Fig 2. Timetable from onset of symptoms to reperfusion. Note that the time to reperfusion from the initial administration of thrombolysis agents was significantly shorter in the pressurecontrolled intermittent coronary sinus occlusion (PICSO) group. CS, coronary sinus; Ad, time of admission; CL, time (in min) of entrance into the catheter laboratory.

\section{Hemodynamic Effect}

Table 3 lists the hemodynamic effects of PICSO. Heart rate, aortic pressure, pulmonary pressure and cardiac index were not significantly affected by PICSO. There was no adverse effect on hemodynamics. Mean systolic CS pressure significantly increased from $8.3 \mathrm{mmHg}$ to $47.4 \mathrm{mmHg}$ when the CS was occluded.

\section{Reperfusion Time}

The mean time to insert a balloon catheter into the CS was $11 \pm 4 \mathrm{~min}$. The time interval to completion of reperfusion in the infarct-related artery from the initial administration of thrombolysis agents was significantly shorter for the

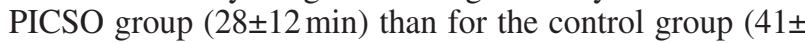
$18 \mathrm{~min}, \mathrm{p}=0.014)$ (Fig 2). The difference of the time interval from the onset of symptoms to reperfusion was not significant between the 2 groups.

\section{Enzymatic Infarct Size}

The PICSO group showed significantly less total CK release than that of the control group (Fig 3). The peak CK activity value was slightly smaller and the peak time of CK was slightly earlier for the PICSO group than those for the control group ( $\mathrm{p}=0.16,0.09$, respectively).

\section{Cardiac Function Analysis}

A left ventriculogram done at 30 days after myocardial infarction (MI) showed that the PISCO group had significantly smaller abnormally contracting segments (21.4 $16.5 \%)$ than the control group $(31.9 \pm 10.6 \%$; $\mathrm{p}<0.05)$. 
Table 4 Serial Changes in Lactate Extraction Ratio

\begin{tabular}{lcccccc}
\hline \hline & $B L$ & Reperfusion & $6 h$ & $12 h$ & $18 h$ & $24 h^{*}$ \\
\hline PICSO & $13.2 \pm 32.9$ & $-1.25 \pm 63.4$ & $17.1 \pm 23$ & $16.1 \pm 24.6$ & $17.6 \pm 25.1$ & $19.1 \pm 26.5$ \\
Control & $-10.2 \pm 38.2$ & $-27.4 \pm 51.9$ & $2.2 \pm 15.7$ & $0.4 \pm 15.3$ & $0.2 \pm 18.9$ & $-8.8 \pm 33.8$ \\
BL-adjusted difference & & $-4.9(11.0)$ & $4.6(4.4)$ & $4.3(4.0)$ & $4.5(3.8)$ & $10.5(5.4)$ \\
\hline
\end{tabular}

$*$ At 24 h, the lactate extraction ratio almost reached statistical significance $(p=0.059)$.

$B L$-adjusted difference (standard error), difference in mean lactate extraction ratio (PICSO-control) adjusted for baseline differences between groups by analysis of covariance. Other abbreviations see in Tables 1,2.

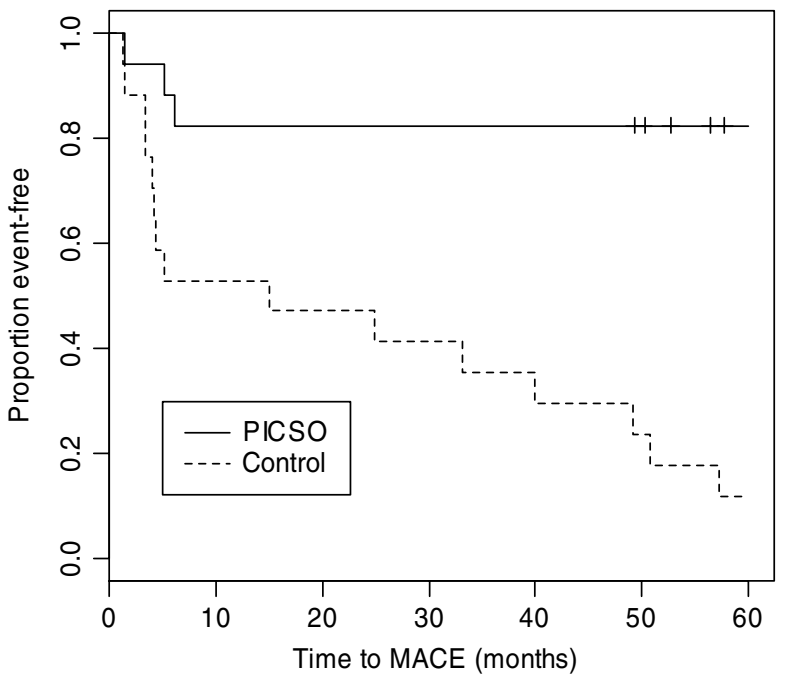

Fig 4. Time to first major adverse cardiovascular events (MACE). After 60 months, a significant difference between the 2 groups was observed $(\mathrm{p}<0.0001)$. PICSO, pressure-controlled intermittent coronary sinus occlusion.

Global and regional ejection fractions, which were measured for each group, failed to show any differences between the PICSO group $(41.3 \pm 17.1,23.2 \pm 17.1$, respectively) and control group ( $49.6 \pm 10,22.8 \pm 17$, respectively).

One patient in the PICSO group, who had a very severe course and had a delayed reperfusion, which is demonstrated by his value for total CK release, had a very low ejection fraction of $8 \%$, and he was among those who needed further intervention. Otherwise, median values showed no difference between groups (51\%), with regional ejection fraction values being $31 \%$ for the PICSO group compared with $26 \%$ for the control group.

The value for defect severity, which was assessed using ${ }^{201} \mathrm{Tl}$ SPECT perfusion at 30 days after onset of symptoms, was significantly smaller for the PICSO group compared with the control group (Fig 3), indicating that PICSO contributed to reducing infarct size. The QRS score for the PICSO group (6.7 \pm 6.7$)$ was also significantly smaller than that for the control group $(11 \pm 4.3, \mathrm{p}=0.038)$.

\section{Myocardial Lactate Metabolism After Thrombolysis}

Lactate extraction became positive within $6 \mathrm{~h}$ from the onset of symptoms in the PICSO group. Conversely, lactate extraction in the control group was still negative or around zero $24 \mathrm{~h}$ after the onset. Baseline-adjusted reference (PICSO-control) demonstrated that there was almost a statistical difference between the groups at $24 \mathrm{~h}$ after reperfusion (Table 4).

\section{Restenosis}

After adjusting for baseline differences, the PICSO group had a lower stenosis value at Day 30. Average baselineadjusted stenosis at Day 30 was $65.5 \%$ for the PICSO group but $73.5 \%$ for the control group $(\mathrm{p}=0.0126)$. In other words, the incidence of stenosis was about $8 \%$ less (SE, $3.02 \%$ ) after PICSO compared with the control group (Table 2). Non-parametric analysis confirmed this result with a p-value of 0.044 .

\section{Time to MACE}

The median follow-up time was 67 months (25th percentile, 58 months; 75th percentile, 81 months). We observed MACE in 15 patients (44\%; 3 patients in the PICSO group, 12 patients in the control group). Progression of the culprit lesion of the proximal LAD was observed in all 15 patients. Four patients in the control group developed reinfarction that was fatal, resulting in 3 cardiac deaths. Recurrence of unstable angina was observed in 8 control patients compared with 3 PICSO patients. In the control group, this resulted in PCI (7 patients) and, because of multivessel involvement in 1 patient, in CABG. Three PICSO patients had PCI only. Cardiac event-free survival was significantly longer in the PICSO group compared with the control group $(\mathrm{p}<0.0001)$. The cardiac event-free survival rate at 48 months was $82 \%$ for the PICSO group but only $23 \%$ for the control group (Fig 4). The relative risk for cardiac events (95\% CI) of PICSO compared with control was 0.12 $(0.03,0.42)$, meaning that the risk for cardiac events was 8.4 times higher for the control group than for the PICSO group. Adjusting for reperfusion time, the relative risk for PICSO compared with control is $0.14(0.04-0.52)$. Adjusting for pain-to-admission time changes, the relative risk for PICSO compared with control only was 0.19 (0.05$0.59)$. The relative risk estimate did not change by more than 0.02 when any of the other risk factors were included in the analysis, suggesting that the minor differences in risk factor exposure in the 2 groups do not affect our conclusions. There is a strong relationship between restenosis and MACE, the Hazards ratio is 24 (per 25\% stenosis), and confidence limits are 1.86-312 ( $\mathrm{p}=0.0149)$. Adjusting for restenosis, the relative risk of PICSO compared with control was $0.11(0.03,0.40)$, meaning that the risk for MACE was 9 times higher for the control group than for the PICSO group.

\section{Time to Reinfarction}

Time to re-MI was significantly longer in the PICSO group compared with the control group $(\mathrm{p}=0.0152)$. After 48 months, $100 \%$ (95\% CI, 81-100\%) of patients in the PICSO group but only 55.6\% (95\% CI, 20.4-80.4\%) in the control group were free from re-MI. The relative risk for re-MI $(95 \% \mathrm{CI})$ of PICSO compared with control was $0.058(0.0004,0.55)$, meaning that the risk for re-MI was 
17 times higher for the control group than for the PICSO group. Adjusting for reperfusion time, the relative risk for PICSO compared with control was 0.039 (0.0003-0.39). Adjusting for pain-to-admission time changes, the relative risk for PICSO compared with control was 0.06 (0.00050.62 ). The relative risk estimate did not change by more than 0.02 when any of the other risk factors were included in the analysis. Particularly, after adjusting for restenosis, the relative risk of PICSO compared with control was $0.035(0.0002,0.45)$, meaning that the risk for reinfarction was 29 times higher for the control group than for the PICSO group.

\section{Discussion}

This re-evaluation of a clinical trial, which was published in this Journal prior to current therapies of MI, is unique in several ways, as it represents a pathophysiologic insight into the ischemic and reperfusion period, which is otherwise unavailable under current clinical standards. Data analyzed should be viewed as an important influx of innovation gained from past experience and as an unique opportunity to view them with today's clinical knowledge and scientific experience.

Immediate Effects and Their Influence on Clinical Outcome

The study's main findings can be differentiated between short- and long-term effects. First, the study showed that PICSO significantly contributed to a reduction in infarct size and to re-establishing myocardial lactate metabolism in the acute phase. Second, reperfusion was achieved earlier in the PICSO group, indicating that the interaction between clot lysis and ischemic microcirculation was more favorable with intermittent pressure elevation in the coronary venous system. The third important finding is the effect of PICSO on the grade of the residual stenosis itself. In terms of the remaining stenosis, the difference between the trends in both groups can be explained by the improved clot lysis and patency of the microcirculation at the time of reperfusion. The results of myocardial infarct size evaluated 30 days after symptom onset (Fig 3 ) also supports the positive effect of PICSO on myocardial salvage. In contrast, there was no indication that global function could be improved with this technique. Whether this technique (ie, extending the PICSO therapy during the reperfusion phase) and state-of-the-art technology will be able to improve myocardial function globally needs further investigation with a larger number of patients. The principle finding of the present study was that generating an impulse for only about $1 \mathrm{~h}$ of PICSO during the ischemic period shortly before reperfusion resulted in a significant reduction in MACE during long-term follow up (Fig 4).

\section{PICSO and Salvage Time}

PICSO also has the potential to achieve earlier recanalization as an adjunct to reperfusion therapy (Fig 2). PICSO increased coronary venous system pressure, resulting in augmented retrograde access to the microcirculation and collateral perfusion in the area at risk. It is reported that PICSO improves washout and that there is less pronounced no flow phenomena in the area at risk? Although the potential to resolve a thrombus seems to be similar in both groups, changes in the impedance to coronary flow might be different in the treated group. PICSO may also enhance the thrombolytic agents' lytic effects on any microthrombi occluding the microcirculation in jeopardized areas.

The earlier and more complete reperfusion in the PICSO group may have affected long-term results. Vasodilatation is indicated to be one of the positive effects of PICSO. In addition, washout seems to be a contributor to clearing the ischemic microcirculation and to protecting its structural integrity.

\section{PICSO and Restenosis After Thrombolysis Therapy}

Clearing the culprit region from residual clots is an important goal in clinical practice. In the pre acute PCI era the residual stenosis after lysis therefore represents also the residual impedance of the reperfused microcirculation. The mean residual infarct artery stenosis in the present study (Table2) was similar that of the conservative strategies group after thrombolysis in the TIMI phase II-A trial (ie, $67.2 \%)^{23}$ The cumulative performance rate of PCI at 1 year was $23.9 \%$ in that group. A German study of AMI patients treated with thrombolysis in the MITRA trial showed that MACE in the thrombolysis group was $32.3 \%$ during a 17month follow up ${ }^{24}$ Henriques and colleagues showed that the combined endpoint of MACE in the patient group treated by streptokinase was $59 \%$ during 8 years of follow up 25 When compared with those large clinical trials, there might be a higher incidence of MACE in the present study's control group (52.9\%) at 17 months, and yet the incidence of MACE tends to be lower in the PICSO group (17.6\% at 60 months; Fig 4) ${ }^{26}$ As indicated below, there is also a relationship between MACE and the occurrence of restenosis.

\section{Regeneration Potential}

Weigel and colleagues showed that the pressure increase in the coronary venous circulation generated by PICSO augmented the local expression of heme oxygenase- 1 and vascular endothelial growth factor (VEGF) in acute ischemia in porcine myocardium?27,28 Seko and colleagues showed that pulsatile mechanical stretch stimulated VEGF secretion by cultured rat cardiac myocytes 29 Pulsatile stretch induces mRNA expression of VEGF and VEGF receptors in cardiac myocytes. Yamamoto and colleagues demonstrated that endothelial progenitor cells (EPCs) were sensitive to shear stress and shear stress stimulated EPC proliferation, suggesting that their vasculogenic activities may be modulated by shear stress? 30

With this in mind we put forward the following PICSO mechanism: during balloon inflation rising coronary sinus pressure "massages" venous endothelium thus inducing periodic pulsatile stretch whereas deflation of the CS balloon introduces a rapid decrease in CS pressure imposing a powerful shear stress during the PICSO cycle. Although our theory cannot explain whether there are structural changes in the reperfused myocardium induced by PICSO, our data suggest that there is another beneficial mechanism involved, which might be the aforementioned mechanism on vasoactive and neoangiogenetic gene expression. Conducting studies supporting our hypothesis will be the basis of our future research.

\section{The Context to Other 'Adjunct Therapies'}

Although reperfusion strategies are the gold standard in modern interventional cardiology, there is still a place for the protection of damaged myocardium. Recent clinical trials show that adenosine or nicorandil as an adjunct therapy attenuated reperfusion injury, $8,9,31$ Moreover, postconditioning using the repetition of brief episodes of ischemia/ 
reperfusion as a 'gentle' temporary reperfusion performed at the time of recanalization after prolonged ischemia, results in significantly reduced infarct size and attenuated reperfusion injury, including the development of oxidants, pro-inflammatory cytokines, neutrophils, and pro-apoptotic regulators 32,33 Postconditioning attenuates cardiomyocyte damage and coronary vascular endothelium injury also reduces apoptosis 33 Focusing on the mechanism of restenosis after AMI, inflammation plays an important role in atherogenesis and atherothrombotic events, 34 and highsensitivity C-reactive protein (hs-CRP) has been associated with an increased risk of coronary artery disease 35 A combination of soft plaques and elevated preprocedural hs-CRP levels are associated with a higher incidence of restenosis in bare-metal stent implantation 36 In this context, PICSO might be a valuable and easily applicable technology.

\section{A Second Window of Opportunity, a Step Towards Clinical Significance and a New Pathophysiologic Insight}

The mainstream of clinical interest passed CSI and especially PICSO despite obvious improvements since the observational study on PICSO and its technology was published in this Journal several years ago. Since then clinical focus has changed towards the emergent technology of acute PCI, and the application of CSI was also hindered because of the problem of how to access the CS securely and rapidly. This hiatus in interest buried the enormous pathophysiologic potential of these interventions. In the present report, we were able to assess the significance of PICSO during the ischemic/reperfusion period far beyond any other adjunct therapy tested clinically. This report also showed convincingly that only $59 \pm 30$ min of treatment was able to set an impulse for long-lasting clinical improvements. Furthermore, we were able to shed light on an otherwise ignored pathophysiologic consequence of an apparent inflammatory process originating in the previous ischemic reperfused myocardial area setting an atherosclerotic stimulus-inducing restenosis. PICSO seems to have a positive effect in changing the microenvironment in the ischemic/reperfused myocardium.

There is, however, also a major obstacle to using PICSO in an acute setting like AMI, as the CS catheterization is cumbersome and needs special attention. Using the CS as an access route for myocardial protection has been routine practice in cardiac surgery. Biventricular pacing via the CS is also a common remedy for patients with chronic congestive hart failure. Not only cardiac surgeons but also cardiologists use the CS in current clinical practice. In the present study, CS catheter insertion time via the basilic or brachial vein was $11 \pm 4 \mathrm{~min}$, without any complications. Present technology and interventional experience must be used to focus on CS access so that the pathophysiologically promised success can be reached.

Presently, we are forced to treat compromised patients (ie, multi-vessels disease with diffuse atherosclerotic lesions and poor runoff and compromised ventricular function). Needless to say, timely reperfusion is the principal goal in improving the survival of patients with AMI. There is, however ample, evidence that PICSO could take the logistic tension from acute PCI as a present gold standard therapy by widening the window for myocardial salvage, influencing the reperfusion process and thus inducing structural changes, which would result in a significant improvement of the clinical outcome and myocardium protection, salvage and regeneration.
In summary, the clinical potential of this technology can also be demonstrated as an adjunct therapy in complicated and even compromised revascularization procedures. If the results described in this report can be duplicated during the ischemic period prior and after acute PCI, the road towards an efficient adjunctive therapy, as well as a modern alternative in myocardial protection, will be available for patients otherwise beyond the therapeutic window, who are not suitable for standard revascularization procedures.

\section{A Word on Behalf of the Authors}

Dr Werner Mohl developed the concept of PICSO and one of us Dr Kazuo Komamura designed and realized the study. Despite the success of our work, each of us were sidetracked from our research on CSI; one going into clinical cardiac surgery and one into heart failure research. Our careers coincided with the hiatus in mainstream clinical and research interest. However, stem cell research stirred our interest again and the results from Weigel et $\mathrm{al}^{27}$ on the induction of angiogenetic genes by PICSO made the present re-evaluation study necessary. There was the concern that the induction of neoangiogenetic genes, as well as other growth factors, might influence restenosis negatively. To our surprise the findings, including the influence on MACE, was quite the opposite. Therefore, the present report, which was structured mainly by Dr Hirofumi Kasahara, is the necessary result of this process. Discussions originating in this evaluation were also the beginning of a completely new perspective of CSI, its technology and pathophysiologic consequences. This culminated presently in formulating a new hypothesis on 'embryonic recall' 28 Hence, we are certain that this report will again stir interest in this important field to protect, regenerate and recover the heart.

\section{References}

1. Nishida T, Shimokawa H, Oi K, Tatewaki H, Uwatoku T, Abe K, et al. Extracorporeal cardiac shock wave therapy markedly ameliorates ischemia-induced myocardial dysfunction in pigs in vivo. Circulation 2004; 110: 3055-3061.

2. Mohl W. The momentum of coronary sinus interventions clinically. Circulation 1988; 77: 6-12.

3. Syeda B, Schukro C, Heinze G, Modaressi K, Glogar D, Maurer G, et al. The salvage potential of coronary sinus interventions: Metaanalysis and pathophysiologic consequences. J Thorac Cardiovasc Surg 2004; 127: 1703-1712.

4. Mohl W, Simon P, Neumann F, Schreiner W, Punzengruber C. Clinical evaluation of pressure-controlled intermittent coronary sinus occlusion: Randomized trial during coronary artery surgery. Ann Thorac Surg 1988; 46: 192-201.

5. Komamura K, Mishima M, Kodama K. Preliminary clinical experience with intermittent coronary sinus occlusion in combination with thrombolytic therapy in acute myocardial infarction. Jpn Circ J 1989; 53: $1152-1163$.

6. Incorvati RL, Tauberg SG, Pecora MJ, Macherey RS, Krucoff MW, Dianzumba SB, et al. Clinical applications of coronary sinus retroperfusion during high risk percutaneous transluminal coronary angioplasty. J Am Coll Cardiol 1993; 22: 127-134.

7. Boekstegers P, Giehrl W, von Degenfeld G, Steinbeck G. Selective suction and pressure-regulated retroinfusion: An effective and safe approach to retrograde protection against myocardial ischemia in patients undergoing normal and high risk percutaneous transluminal coronary angioplasty. J Am Coll Cardiol 1998; 31: 1525-1533.

8. Mangano DT, Miao Y, Tudor IC, Dietzel C. Post-reperfusion myocardial infarction: Long-term survival improvement using adenosine regulation with acadesine. J Am Coll Cardiol 2006; 48: 206-214.

9. Ota S, Nishikawa H, Takeuchi M, Nakajima K, Nakamura T, Okamoto $\mathrm{S}$, et al. Impact of nicorandil to prevent reperfusion injury in patients with acute myocardial infarction: Sigmart Multicenter Angioplasty Revascularization Trial (SMART). Circ J 2006; 70: 1099-1104.

10. Patel AN, Geffner L, Vina RF, Saslavsky J, Urschel HC Jr, Kormos 
$\mathrm{R}$, et al. Surgical treatment for congestive heart failure with autologous adult stem cell transplantation: A prospective randomized study. J Thorac Cardiovasc Surg 2005; 130: 1631-1638.

11. Chesebro JH, Knatterud G, Roberts R, Borer J, Cohen LS, Dalen J, et al. Thrombolysis in Myocardial Infarction (TIMI) Trial, Phase I: A comparison between intravenous tissue plasminogen activator and intravenous streptokinase: Clinical findings through hospital discharge. Circulation 1987; 76: 142-154.

12. Shell WE, Kjekshus JK, Sobel BE. Quantitative assessment of the extent of myocardial infarction in the conscious dog by means of analysis of serial changes in serum creatine phosphokinase activity. J Clin Invest 1971; 50: 2614-2625.

13. Norris RM, Whitlock RM, Barratt-Boyes C, Small CW. Clinical measurement of myocardial infarct size. Modification of a method for the estimation of total creatine phosphokinase release after myocardial infarction. Circulation 1975; 51: 614-620.

14. Feild BJ, Russell RO Jr, Dowling JT, Rackley CE. Regional left ventricular performance in the year following myocardial infarction. Circulation 1972; 46: 679-689.

15. Gelberg HJ, Brundage BH, Glantz S, Parmley WW. Quantitative left ventricular wall motion analysis: A comparison of area, chord and radial methods. Circulation 1979; 59: $991-1000$.

16. Prigent F, Maddahi J, Garcia EV, Satoh Y, Van Train K, Berman DS. Quantification of myocardial infarct size by thallium-201 singlephoton emission computed tomography: Experimental validation in the dog. Circulation 1986; 74: 852-861.

17. Caldwell JH, Williams DL, Harp GD, Stratton JR, Ritchie JL. Quantitation of size of relative myocardial perfusion defect by single-photon emission computed tomography. Circulation 1984; 70: 1048-1056.

18. Wagner GS, Freye CJ, Palmeri ST, Roark SF, Stack NC, Ideker RE, et al. Evaluation of a QRS scoring system for estimating myocardial infarct size. I: Specificity and observer agreement. Circulation 1982; 65: $342-347$

19. Heinze G, Gnant M, Schemper M. Exact log-rank tests for unequal follow-up. Biometrics 2003; 59: 1151-1157.

20. Heinze G, Schemper M. A solution to the problem of monotone likelihood in Cox regression. Biometrics 2001; 57: 114-119.

21. Vickers AJ, Altman DG. Statistics notes: Analysing controlled trials with baseline and follow up measurements. BMJ 2001; 323: $1123-$ 1124 .

22. Berger PB, Ellis SG, Holmes DR Jr, Granger CB, Criger DA, Betriu A, et al. Relationship between delay in performing direct coronary angioplasty and early clinical outcome in patients with acute myocardial infarction: Results from the global use of strategies to open occluded arteries in Acute Coronary Syndromes (GUSTO-IIb) trial. Circulation 1999; 100: 14-20.

23. Rogers WJ, Baim DS, Gore JM, Brown BG, Roberts R, Williams $\mathrm{DO}$, et al. Comparison of immediate invasive, delayed invasive, and conservative strategies after tissue-type plasminogen activator: Results of the Thrombolysis in Myocardial Infarction (TIMI) Phase IIA trial. Circulation 1990; 81: 1457-1476.

24. Zhan R, Schiele R, Schneider S, Gitt AK, Heer T, Wienbergen H, et al. Long-term follow-up of patients with acute myocardial infarction treated with primary angioplasty or thrombolysis: Results of the MITRA trial. Z Kardiol 2002; 91: 49-57 (in German with English abstract).

25. Henriques JP, Zijlstra F, van't Hof AW, de Boer MJ, Dambrink JH, Gosselink AT, et al. Primary percutaneous coronary intervention versus thrombolytic treatment: Long term follow up according to infarct location. Heart 2006; 92: 75-79.

26. Kasahara H, Komamura K, Glogar D, Kodama K, Hirayama A, Mohl W. Do long term effects after PICSO endorse the potential of cardiac regeneration after acute myocardial infarction? Wien Klin Wochenschr 2007; 119(Suppl): 23-26.

27. Weigel G, Kajgana I, Bergmeister H, Riedl G, Glogar HD, Gyongyosi $\mathrm{M}$, et al. Beck and back: A paradigm change in coronary sinus interventions-pulsatile stretch on intact coronary venous endothelium. J Thorac Cardiovasc Surg 2007; 133: 1581-1587.

28. Mohl W. Embryonic recall: Myocardial regeneration beyond stem cell transplantation. Wien Klin Wochenschr 2007; 119: 333-336.

29. Seko Y, Takahashi N, Shibuya M, Yazaki Y. Pulsatile stretch stimulates vascular endothelial growth factor (VEGF) secretion by cultured rat cardiac myocytes. Biochem Biophys Res Commun 1999; 254: $462-465$.

30. Yamamoto K, Takahashi T, Asahara T, Ohura N, Sokabe T, Kamiya A, et al. Proliferation, differentiation, and tube formation by endothelial progenitor cells in response to shear stress. J Appl Physiol 2003; 95: $2081-2088$

31. Mahaffey KW, Puma JA, Barbagelata NA, DiCarli MF, Leesar MA, Browne KF, et al. Adenosine as an adjunct to thrombolytic therapy for acute myocardial infarction: Results of a multicenter, randomized, placebo-controlled trial: The Acute Myocardial Infarction STudy of ADenosine (AMISTAD) trial. J Am Coll Cardiol 1999; 34: $1711-1720$.

32. Zhao ZQ, Corvera JS, Halkos ME, Kerendi F, Wang NP, Guyton RA, et al. Inhibition of myocardial injury by ischemic postconditioning during reperfusion: Comparison with ischemic preconditioning. Am J Physiol Heart Circ Physiol 2003; 285: H579-H588.

33. Vinten-Johansen J, Yellon DM, Opie LH. Postconditioning: A simple, clinically applicable procedure to improve revascularization in acute myocardial infarction. Circulation 2005; 112: 2085-2088.

34. Yip HK, Youssef AA, Chang LT, Yang CH, Sheu JJ, Chua S, et al. Association of interleukin-10 level with increased 30-day mortality in patients with ST-segment elevation acute myocardial infarction undergoing primary coronary intervention. Circ J 2007; 71: 10861091 .

35. Libby P, Ridker PM. Inflammation and atherosclerosis: Role of Creactive protein in risk assessment. Am J Med 2004; 116(Suppl 6A): $9 \mathrm{~S}-16 \mathrm{~S}$.

36. Hong YJ, Jeong MH, Lim SY, Lee SR, Hong SN, Kim KH, et al. Relation of soft plaque and elevated preprocedural high-sensitivity C-reactive protein levels to incidence of in-stent restenosis after successful coronary artery stenting. Am J Cardiol 2006; 98: 341-345. 